\title{
Histidine-rich glycoprotein inhibits the antiangiogenic effect of thrombospondin-1
}

\author{
Ronit Simantov, Maria Febbraio, René Crombie, Adam S. Asch, Ralph L. Nachman, \\ and Roy L. Silverstein \\ Division of Hematology-Oncology, Department of Medicine, Weill Medical College of Cornell University, \\ New York, New York, USA \\ Address correspondence to: Ronit Simantov, Weill Medical College of Cornell University, Room C-606, 1300 York Avenue, \\ New York, New York 10021, USA. Phone: (212) 746-2060; Fax: (212) 746-8866; E-mail: rsimant@mail.med.cornell.edu.
}

Received for publication December 3, 1999, and accepted in revised form November 14, 2000.

\begin{abstract}
Angiogenesis is critical for the growth and proliferation of tumors as well as for normal development. We now describe a novel role for histidine-rich glycoprotein (HRGP) in the modulation of angiogenesis. HRGP is a plasma protein that circulates in relatively high concentrations $(1.5 \mu \mathrm{M})$, but has no known function in vivo. We have shown previously that HRGP binds with high affinity to thrombospondin-1 (TSP-1), a homotrimeric glycoprotein that is a potent inhibitor of angiogenesis. The antiangiogenic activity of TSP-1 is mediated by the binding of properdin-like type I repeats to the receptor CD36. We found that binding of HRGP to TSP-1 was similarly mediated by TSP type I repeats. HRGP colocalized with TSP-1 in the stroma of human breast cancer specimens, and this interaction masked the antiangiogenic epitope of TSP-1. In assays performed in vitro of endothelial cell migration and tube formation, and in vivo corneal angiogenesis assays, HRGP inhibited the antiangiogenic effect of TSP-1. These studies suggest that HRGP can modulate the antiangiogenic activity of TSP-1, and identify a potential mechanism of resistance to the antiangiogenic effect of TSP-1.
\end{abstract}

J. Clin. Invest. 107:45-52 (2001).

\section{Introduction}

Angiogenesis, the development of new blood vessels, is necessary for a variety of normal physiologic processes as well as for the growth and proliferation of tumors. The identification of natural modulators of angiogenesis is essential to the understanding of this complex process, and may provide attractive targets for therapeutic intervention. Histidine-rich glycoprotein (HRGP) is a plasma protein with an unusually high histidine and proline content that circulates in relatively high concentrations $(1.5 \mu \mathrm{M})$, but has no known function in vivo (1). Several in vitro interactions of HRGP have been described, such as binding to metals, heparin, and several proteins including plasminogen, fibrinogen, and thrombospondin-1 (TSP-1) $(2,3)$. Surface-bound HRGP can accelerate the activation of plasminogen by tissue plasminogen activator, suggesting a role for HRGP in the fibrinolytic system (4). Although there are reports of inherited HRGP polymorphisms and elevated HRGP levels in families with thromboembolic disease, the role of HRGP in thrombosis and fibrinolysis in humans has not been determined (5).

We have shown previously that HRGP binds with high affinity to TSP-1 (6), a 450-kDa homotrimeric adhesive glycoprotein that is a potent inhibitor of angiogenesis (7-9). TSP-1 is secreted by activated platelets and a variety of normal vascular cells, including endothelial and smooth muscle cells (10), and has been shown to inhibit endothelial cell (EC) proliferation, migration, and tube formation in response to multiple angiogenic stimuli (11). The antiangiogenic activity of TSP-1 has been localized to the properdin-like type I repeats, and synthetic peptides derived from the type I domains have been found to have potent antiangiogenic activity in vivo and in assays of EC function (9).

Although TSP-1 interacts with a number of distinct cellular receptors, $\mathrm{CD} 36$ has been recognized as the critical angiostatic receptor for TSP-1 $(12,13)$. The binding of TSP-1 to CD36 is mediated by the peptide sequence cysteine-serine-valine-threonine-cysteine-glycine (CSVTCG), the same type I repeat shown to have antiangiogenic activity (14). We have now explored the structure-function relationships that mediate interactions between TSP-1 and HRGP, and report that the binding of HRGP is localized to the type I repeats, the same sequence motifs responsible for CD36 binding. Moreover, we now report the role of HRGP in inhibiting the antiangiogenic activity of TSP-1.

\section{Methods}

Reagents. Recombinant human bFGF was purchased from Research Diagnostics Inc. (Flanders, New Jersey, USA). Rabbit Ab to HRGP was kindly supplied by Lawrence Leung, Stanford University, Palo Alto, California, USA. TSP-1 was purified from human platelet releasate by heparin affinity and anion 
exchange chromatography on Mono Q-Sepharose (Pharmacia Biotech Inc., Piscataway, New Jersey, USA) as described (15). Radiolabeling was performed with $\mathrm{Na}^{125}$ I (Amersham Life Sciences Inc., Arlington Heights, Illinois, USA) using immobilized chloramine $\mathrm{T}$ (IodoBeads; Pierce Chemical Co., Rockford, Illinois, USA) as described (16). Fusion proteins (FPs) consisting of CD36 and glutathione $S$-transferase (GST) have been described previously (17). Recombinant TSP peptides were a gift of Jeffrey Laurence (Weill Medical College of Cornell University). HRGP was purified from human plasma by lys-plasminogen affinity chromatography as described (6). Purified proteins were incubated with polymyxin B-coated agarose (Sigma Chemical Co., St. Louis, Missouri, USA) to remove any potentially contaminating LPS before use in cellular assays. Specific rabbit Ab to CSVTCG was generated by subcutaneous immunization with keyhole limpet hemocyanin-coupled peptide. IgG was purified from serum by protein A chromatography (Pierce Chemical Co.). Bovine heparin was purchased from Sigma Chemical Co.

Amino acid sequence alignment. Sequence homology analysis was performed using BLAST-enhanced alignment utility search (BEAUTY, SIM, ALIGN, and LALIGN) via search engines accessed through http://dot.imgen.bcm.tmc.edu $(18,19)$.

Cell culture. Human dermal microvascular ECs (HMVECs) were purchased from Cascade Biologics Inc. (Portland, Oregon, USA) and maintained in Medium 131 with $5 \%$ commercial Microvascular Growth Supplement (MVGS). CD36 expression, which was confirmed by flow cytometric analysis using a murine anti-human CD36 mAb (FA6) and a fluorescein-conjugated goat anti-mouse IgG (PharMingen, San Diego, California, USA), was maintained through passage 5.

ELISA was performed as described (6). Briefly, TSP$1(4 \mu \mathrm{g} / \mathrm{ml})$ in coating buffer $(0.5 \mathrm{M}$ sodium carbonate, $\mathrm{pH}$ 9.6) was applied to microtiter plates (Becton Dickinson Immunocytometry Systems, Lincoln Park, New Jersey, USA) for 3 hours at $37^{\circ} \mathrm{C}$. In some assays, plates were coated with murine $\mathrm{mAb}$ to TSP clone 11.4 (15) in carbonate buffer overnight, then washed with wash buffer $(0.05 \%$ Tween in $20 \mathrm{mM}$ Tris, and $150 \mathrm{mM} \mathrm{NaCl}, \mathrm{pH} 7.5)$ and incubated with TSP-1 (4 $\mu \mathrm{g} / \mathrm{ml}$ ) for 2 hours (16). Plates were then washed with wash buffer $(0.05 \%$ Tween in $20 \mathrm{mM}$ Tris with 150 $\mathrm{mM} \mathrm{NaCl}, \mathrm{pH} 7.5)$ and blocked for 1 hour at $37^{\circ} \mathrm{C}$ with $1 \%$ BSA in Tris-Tween. Varying amounts of HRGP with and without peptide were added, and plates were incubated for 18 hours at $4^{\circ} \mathrm{C}$. After washing, alkaline phosphatase-conjugated anti-HRGP or nonimmune rabbit $\mathrm{Fab}^{\prime} 2$ was added. This was incubated for 3 hours at $37^{\circ} \mathrm{C}$, after which $p$-nitrophenyl phosphate substrate was added and OD was read in a microplate reader at $405 \mathrm{~nm}$ (Molecular Devices Corp., Menlo Park, California, USA).

Solid-phase binding assays were performed as described (16). Briefly, HRGP in carbonate buffer was
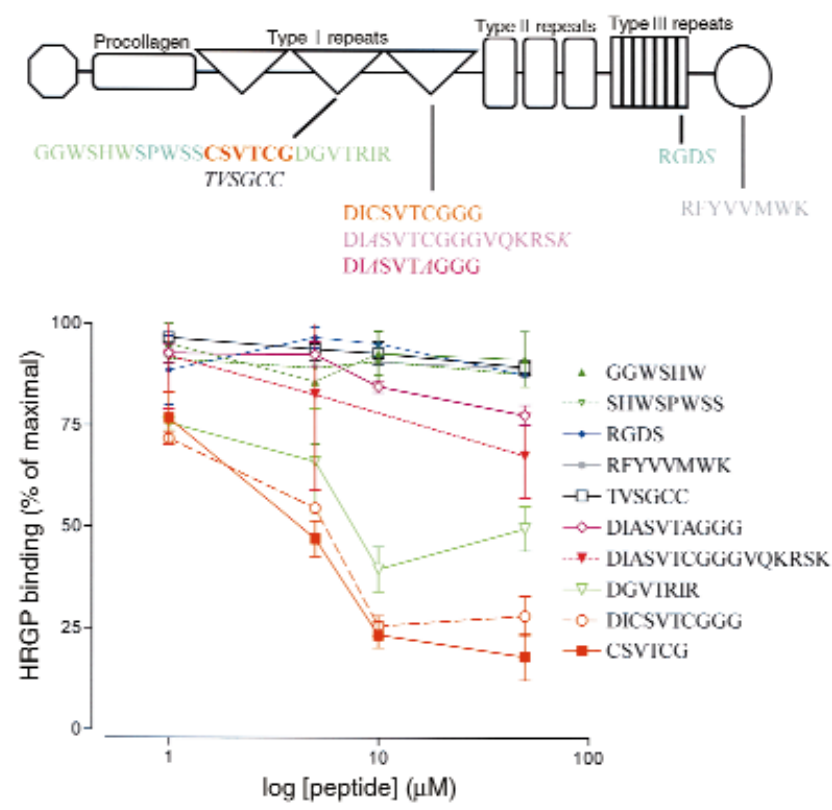

\section{Figure 1}

The type 1 repeat ofTSP mediates binding to HRGP. Peptides derived from the multiple domains ofTSP-1 are shown. Mutated amino acids are italicized and type I repeat-derived peptides are in boldface. HRGP $(1 \mu \mathrm{g} / \mathrm{ml})$ binding to TSP-1 immobilized on plastic wells was measured by enzyme-linked immunosorbent assay (ELISA) using an alkaline phosphatase-conjugated anti-HRGP Ab and p-nitrophenyl phosphate substrate. HRGP binding in the presence of TSP- 1 peptides is expressed as percent binding in the absence of peptide.

immobilized on 96-well strips (Immulon 4 Removawell; Dynatech Laboratories Inc., Burlington, Massachusetts, USA) overnight at $4^{\circ} \mathrm{C}$. After blocking with $1 \% \mathrm{BSA}$, ${ }^{125}$ I-TSP-1 was incubated in the presence of varying concentrations of CD36 FP in triplicate, and bound radioactivity was quantified on a gamma counter. Nonspecific binding was determined by carrying out binding in the presence of excess unlabeled ligand.

Tube formation assay. Early passage (passage 2 or passage 3) HMVECs in $0.2 \%$ MVGS were grown on $150 \mu \mathrm{l}$ of Matrigel (Becton Dickinson Labware, Bedford, Massachusetts, USA) on 48-well plates at $3 \times 10^{4}$ cells per well. Reagents were added and cells were incubated in an atmosphere of $5 \% \mathrm{CO}_{2}$ at $37^{\circ} \mathrm{C}$ for 24 hours, then photographed. Images were scanned and the number of branched structures was counted using Scion Image software (Scion Corp., Frederick, Maryland, USA).

EC chemotaxis assays were performed as described using a modified Boyden chamber with $8-\mu \mathrm{m}$ gelatincoated membranes (20). Briefly, confluent flasks of HMVECs were grown in $0.1 \%$ MVGS overnight, and plated at $3 \times 10^{4}$ cells per well on a gelatinized porous membrane (Osmonics Inc., Minnetonka, Minnesota, USA). Reagents were added to wells, and cells were allowed to migrate in an atmosphere of $5 \% \mathrm{CO}_{2}$ at $37^{\circ} \mathrm{C}$ for 4 hours. Membranes were stained, and the number of migrating cells was counted. Results were expressed as the percentage of cells migrating toward 
bFGF (38-137 cells per 10 high-power fields) minus the number of cells migrating in the absence of bFGF (4-14 cells per 10 high-power fields).

In vivo Matrigel plug assays were performed as described (21). Briefly, $500 \mu \mathrm{l}$ of Matrigel mixed with bFGF (10 ng/ml), TSP-1 (100 ng/ml), and/or HRGP $(50 \mathrm{ng} / \mathrm{ml})$ was injected subcutaneously near the abdominal midline of C57BL/6 mice. Gels were removed after 10 days, fixed in $1 \%$ paraformaldehyde, and embedded in paraffin. Blocks were then sectioned, and stained with hematoxylin and eosin.

Immunohistochemistry. Immunohistochemistry of Matrigel specimens was performed on unstained sections using an anti-human von Willebrand factor (vWF) IgG (DAKO A/S, Glostrup, Denmark) or isotype-matched control (Sigma Chemical Co.) and a biotin-streptavidin-peroxidase $\mathrm{Ab}$ and development system (Vector Laboratories Inc., Burlingame, California, USA) as described (22). Specimens were counterstained with Mayer's hematoxylin (InnoGenex, San Ramon, California, USA). After scanning, the degree of angiogenesis was determined by counts of vWF-positive blood vessels using Scion Image. For breast tissue, frozen sections of freshly obtained human breast carcinoma were incubated with rabbit $\mathrm{Ab}$ to HRGP or CSVTCG, murine mAb to TSP, or isotype-matched controls (Sigma Chemical Co.), and developed as above.

Corneal angiogenesis assays. Pellets composed of hydron (Hydro Med Sciences, Cranbury, New Jersey, USA) and sucralfate (Teva Pharmaceuticals, North Wales, Pennsylvania, USA) were mixed with proteins or growth factors, and were prepared and implanted as described (23) into the corneas of wild-type C57BL/6 mice or CD36-null mice backcrossed four times to C57BL/6 mice. After 5 days, eyes were viewed under a dissecting microscope and photographed with a Sony DKC-1000 digital camera. The area of neovascularization was calculated by measuring vessel length from limbus toward the pellet, and the number of clock hours of vascularization, as described $(24,25)$. Studies were approved by the Institutional Animal Use Committee of Weill Medical College of Cornell University.

\section{Results}

The type 1 repeat of TSP-1 mediates binding to HRGP. Previous studies have shown that HRGP binds to TSP-1 saturably, reversibly, and with high affinity $(7 \mathrm{nM})$ (6). We performed binding studies in the presence of peptides derived from the second and third type I repeats, regions to which the antiangiogenic activity of TSP-1 has been localized (26). As shown in Figure 1, HRGP binding to TSP-1 was inhibited by the peptide CSVTCG, but not by scrambled peptide. A CSVTCGcontaining peptide from the third type 1 repeat had similar inhibitory activity, but mutated peptides with substitution of alanine for the cysteine residues in the CSVTCG motif did not inhibit HRGP binding to TSP-1. Binding studies were repeated using TSP-1 bound to immobilized anti-TSP Ab with equivalent results. As an additional control, we measured the binding of plasminogen to TSP-1, and found that plasminogen binding was not inhibited by the CSVTCG peptide, indicating that this interaction was not mediated by the type I repeats.

As further evidence for the specificity of this binding activity, we found that binding of HRGP to TSP- 1 was not inhibited by peptides derived from the TGF- $\beta$ binding sequence (GGWSHW), heparin-binding sequence (SHWSPWSS), the COOH-terminal domain (RFYVVMWK), or an RGDS peptide derived from the type 3 repeats. Recent data has shown that a GVQXR motif, located toward the $\mathrm{COOH}$-terminus relative to the CSVTCG motif in the type I repeats, with an additional similar GVQXR sequence in the procollagen domain, binds to CD36 and contains antiangiogenic activity $(13,27)$. In our studies, the peptide DGVTRIR from the second type I repeat inhibited TSP-1-HRGP binding, suggesting that HRGP may compete with
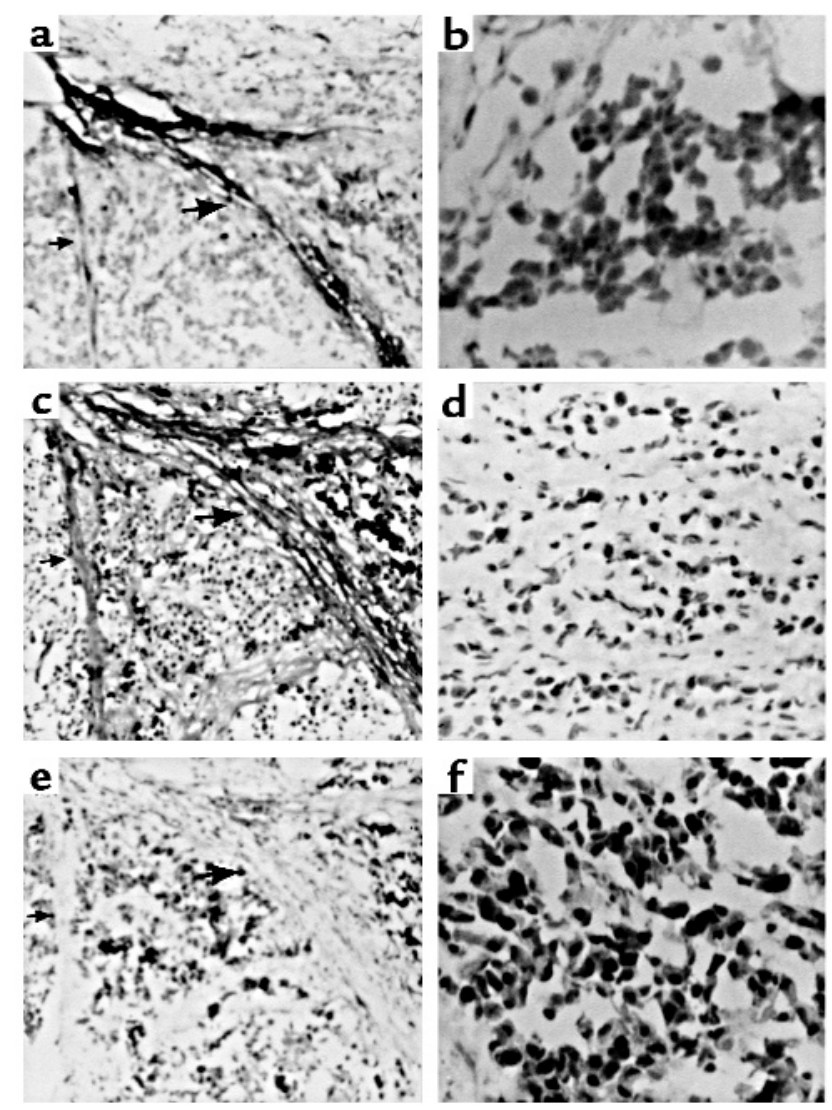

\section{Figure 2}

TSP-1 and HRGP colocalize in stroma of human breast carcinoma. Frozen sections of freshly obtained tumor were incubated with $\mathrm{mAb}$ to TSP-1 ( $\mathbf{a}$ and $\mathbf{b}$ ), polyclonal anti-HRGP ( $\mathbf{c}$ and $\mathbf{d}$ ), or antiserum to CSVTCG (e and $\mathbf{f}$ ). Slides were developed with a peroxidase-conjugated avidin-biotin second Ab system. Adjacent sections show stromal connective tissue bands (arrows) staining with anti-TSP (a) and anti-HRGP $(\mathbf{c})$, but not anti-CSVTCG $(\times 200)(\mathbf{e})$. Tumor-cell staining occurs with anti-TSP $(\mathbf{b})$ and anti-CSVTCG (f) but not with anti-HRGP $(\times 500)(\mathbf{d})$. 


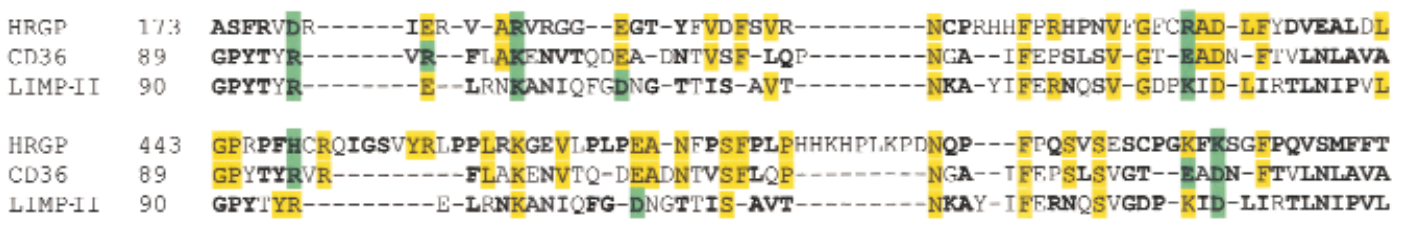

\section{Figure 3}

HRGP contains CLESH-1 homology motifs. Amino acid sequence alignment of CD36/LIMP-II TSP-binding motifs with homologous sequences in HRGP. Results of pattern-based search (BEAUTY) using CD36 exon 5 coding region (CD36 amino acids 95-143) as query identified a split CLESH-1 motif in HRGP (amino acids 443-517). Optimization of alignments using SIM, ALIGN, and LALIGN programs also identified additional repeating motifs (shown, amino acids 173-231). Amino acids that are identical in HRGP and either CD36 or LIMP-II are highlighted in yellow. Green highlights represent conservative substitutions with basic (KRH), acidic (DE), or charged (KRH, DE) amino acids; bold residues represent conserved aromatic (YFW), aliphatic (AG), short-chain (GA, STP), hydrophobic (AGP, IVL, FM), polar/hydrophilic (ST, KRH, DNEQ, CWY), nonpolar/branched (IVL), or hydroxyl (STY) amino acids. GenBank/EMBL accession numbers: huHRGP, P04196; huCD36, M24795; and huLIMPII, D12676.

CD36 for binding of both CSVTCG and flanking sequence. However, an alanine-substituted peptide derived from the third type I repeat (9), which also contained the flanking $\mathrm{COOH}$-terminal sequence GVQKR, had minimal inhibitory activity, suggesting that the cysteine residues in the type I repeat are essential for the interaction of TSP-1 with HRGP.

Although the heparin-binding domain of TSP-1 was recently reported to bind polyhistidine motifs (28), we found that HRGP binding to TSP-1 was not inhibited by the addition of heparin. Studies done at both $\mathrm{pH}$ 7.4 and $\mathrm{pH}$ 7.0, at which HRGP optimally binds heparin, showed that $90 \%$ of maximal binding of HRGP to TSP- 1 was seen in the presence of $100 \mu \mathrm{g} / \mathrm{ml}$ heparin, corroborating our previous findings (6). As further evidence that the TSP-1-HRGP interaction is mediated by the TSP type I repeats, we found that binding of radiolabeled HRGP to TSP-1 that had been resolved on SDS-PAGE and transferred to nitrocellulose was significantly decreased in the presence of antiCSVTCG antiserum, and was completely abolished by the CSVTCG peptide (50 $\mu \mathrm{M}$; not shown).

TSP-1 and HRGP colocalize in vivo. To determine if there is in vivo evidence for TSP-1-HRGP interactions, we performed immunohistochemical studies on serial sections of human breast cancer specimens. TSP- 1 is abundantly expressed in breast tissue, particularly in the stroma and the basement membrane associated with malignant ductal epithelium $(6,29-31)$. We used a murine $\mathrm{mAb}$ to TSP-1 and a rabbit polyclonal antiHRGP Ab to determine the localization of TSP- 1 and HRGP. As shown in Figure 2, both TSP-1 (Figure 2a) and HRGP (Figure 2c) were detected in the stromal connective tissue. Adjacent epithelial cells expressed TSP-1 (Figure 2b); however, HRGP was not detected intracellularly (Figure 2d).

To explore whether the TSP type 1 repeat is involved in TSP-1-HRGP interactions in the breast cancer stroma, we developed a specific $\mathrm{Ab}$ to the CSVTCG peptide. The antiserum was reactive to plasmodium falciparum circumsporozoite protein (known to contain the peptide), and to purified
TSP-1, by Western blot (not shown). Figure 2 shows that the CSVTCG epitope was detectable intracellularly in the breast cancer cells where TSP-1 was detectable but HRGP was absent (Figure 2f). However, in the tumor stroma where HRGP colocalized with TSP-1 (Figure 2e), there was no detectable CSVTCG reactivity. This provides evidence that TSP-1 associates with HRGP in vivo, and that this interaction masks the type I epitope of TSP-1.

HRGP contains CLESH-1 homology motifs. We have previously demonstrated that the binding site for TSP-1 on CD36 and other proteins is defined by homologous, evolutionarily conserved amino acid motifs known as CLESH-1 $(17,32,33)$. As shown in Figure 3, sequence alignments of deduced amino acid sequences of HRGP identified a region (amino acids 443-517) with significant homology to the CLESH-1 domain of CD36 and the CD36-related protein lysosomal integral membrane protein II (LIMP-II) (31\% identity, 74\% similarity) (32). Additional repeating motifs were also identified, including amino acids 173-231 (20\% identity, $70 \%$ similarity). We predicted, therefore, that a CLESH-1 recognition sequence mediates the interaction of HRGP with TSP-1, and that HRGP can interfere with the binding of TSP- 1 to CD36.

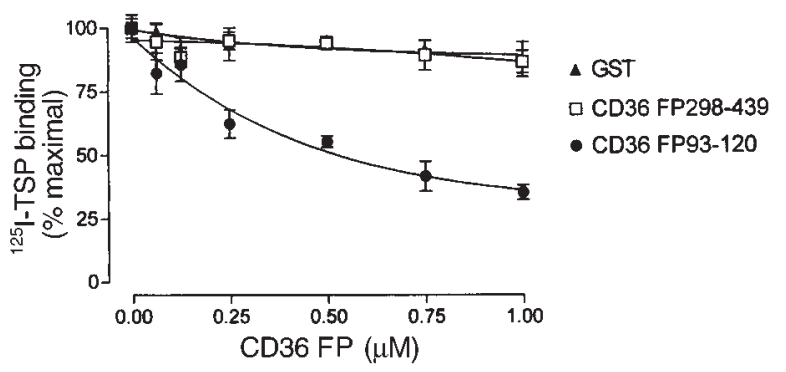

Figure 4

TSP-1 binding of HRGP: competition with CD36 FP93-120. Binding of ${ }^{125}$-TSP-1 to immobilized HRGP was inhibited in the presence of increasing concentrations of CD36 FP93-120, which binds to TSP1, but not in the presence of either GST or CD36 FP298-439, neither of which binds to TSP-1. 


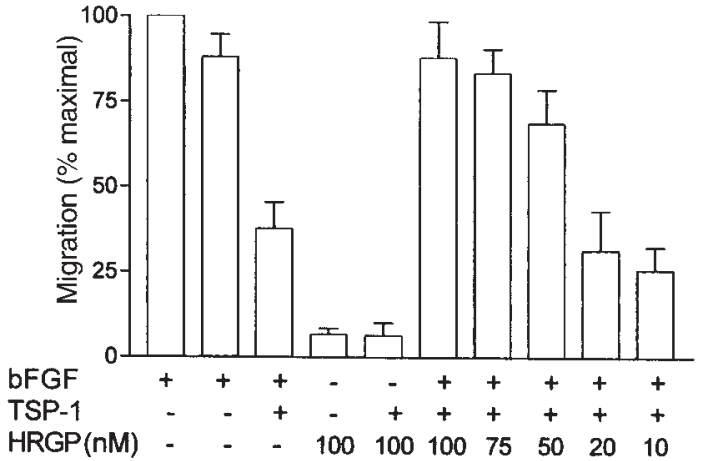

\section{Figure 5}

HRGP inhibits the anti-chemotactic effect of TSP-1. EC migration toward bFGF was measured using a modified Boyden chamber with an $8-\mu \mathrm{m}$ gelatin-coated membrane in the presence and absence of TSP-1 (2 nM) and increasing concentrations of HRGP (0-100 nM). Data from three separate experiments are expressed as a percentage of migration in the presence of bFGF alone. Bars represent SE.

We next performed solid-phase assays in which we measured the binding of radiolabeled TSP-1 to HRGP. As shown in Figure 4, ${ }^{125} \mathrm{I}-\mathrm{TSP}-1$ binding to HRGP was inhibited by increasing concentrations of CD36 FP93-120, a CD36-GST fusion protein that has been shown to bind to TSP-1 $(13,17)$. Similar concentrations of GST alone or CD36 FP298-439 (neither of which binds TSP-1) had no effect.

HRGP inhibits the antiangiogenic activity of TSP-1. The process of angiogenesis requires distinct cellular activities, including migration, proliferation, and differentiation of ECs into capillaries. TSP-1 inhibits EC differentiation in response to multiple angiogenic stimuli (11). We have shown that the antiangiogenic activity of TSP-1 is mediated by interaction of the type I TSP repeat with CD36, its high-affinity receptor on microvascular ECs (13). Because the type I repeat also mediates the interaction of TSP-1 with HRGP, we studied the effect of HRGP on angiogenesis. We measured migration of CD36-expressing microvascular ECs toward bFGF. As shown in Figure 5, the addition of TSP-1 inhibited bFGF-induced migration of ECs. The addition of HRGP inhibited the antichemotactic effect of TSP-1, restoring migration to a level that was $70-80 \%$ of that seen with bFGF alone. As a model for endothelial differentiation, we studied branched tube formation in Matrigel. As shown by others (13), we found that 2 nM TSP- 1 inhibited bFGF-induced (2 $\mathrm{ng} / \mathrm{ml}$ ) tube formation by CD36-expressing microvascular ECs grown on Matrigel under low serum concentrations. The addition of $15 \mathrm{nM}$ HRGP restored tube formation to $88 \pm 11 \%$ of tubes per high-power field seen in EC treated with bFGF alone. HRGP alone, however, did not directly induce tube formation (not shown).

To explore whether HRGP blocks the antiangiogenic activity of TSP-1 in vivo, we performed the corneal angiogenesis assay in which hydron/sucralfate pellets containing bFGF, TSP-1, and/or HRGP were implanted in the corneas of C57BL/ 6 mice. After 5 days, vigorous outgrowth of blood vessels was seen in 13 of 13 eyes implanted with pellets containing $50 \mathrm{ng}$ bFGF, and in only 2 of 11 eyes implanted with pellets containing both bFGF and TSP-1 (200 ng) (Figure 6). In mice implanted with pellets containing bFGF, TSP-1, and HRGP (100 ng, HRGP/TSP-1 molar ratio 3:1), angiogenesis was seen in 10 of 10 eyes $(P=0.02$, $\chi$-square analysis), with an area of neovascularization that was $82.5 \%$ that seen with bFGF alone (Table 1 ). Similar results were obtained when HRGP was added in a pellet separate from bFGF and TSP-1.

Although HRGP alone did not induce angiogenesis in corneal pocket assays, the angiogenic activity of bFGF was significantly increased in the presence of HRGP. As seen in Figure 7, 50 ng HRGP increased the potency of lower concentrations of bFGF, resulting in significantly larger areas of neovascularization at 2.5-10 ng bFGF. Also shown in Figure 7 is the result of experiments demonstrating that HRGP did not affect sensitivity to low concentrations of bFGF in CD36null mice. In fact, the dose-response curve of bFGF in wild-type mice in the presence of HRGP was similar to that seen with bFGF alone in the CD36-null mice. These observations suggest that the increased sensitivity to bFGF in the CD36-null mice, and in wild-type mice in the presence of HRGP, is due to loss of endoge-
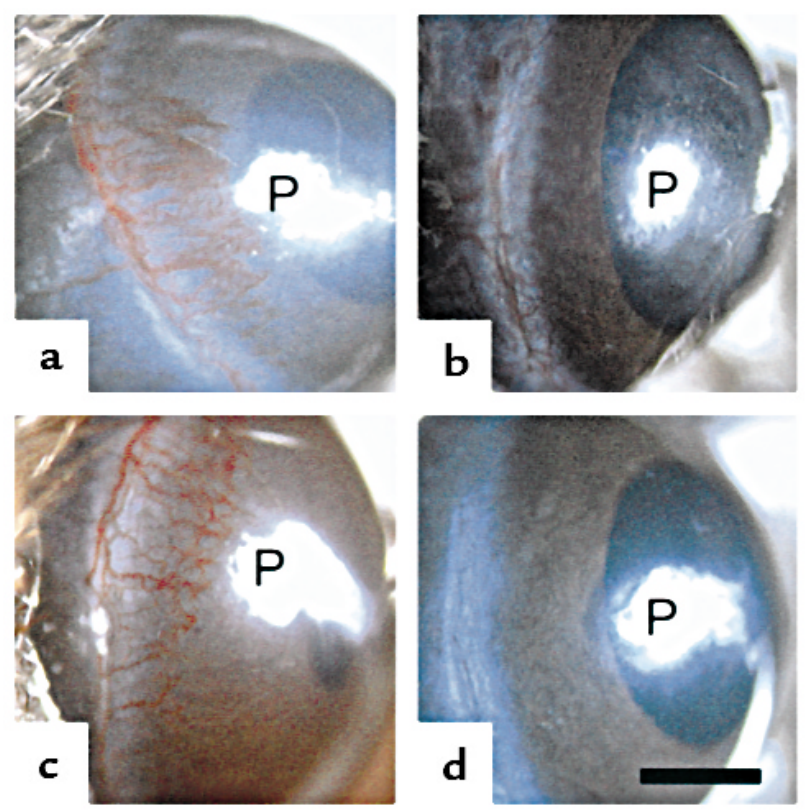

\section{Figure 6}

HRGP inhibits the antiangiogenic effect of TSP-1 in vivo. Hydron/sucralfate pellets containing (a) bFGF (50 ng) (b) bFGF + TSP-1 (200 ng) (c) bFGF + TSP-1 + HRGP (100 ng), or (d) HRGP were implanted in the corneas of $\mathrm{C} 57 \mathrm{BL} / 6$ mice and photographed after 5 days. Representative images are shown. (a) Vigorous neovascularization from the limbus toward the pellet $(\mathrm{P})$ induced by bFGF is shown. The inhibition of angiogenesis by TSP-1 (b) was blocked by the addition of HRGP (c), whereas HRGP alone has no effect (d). Bar, $1 \mathrm{~mm}$. 


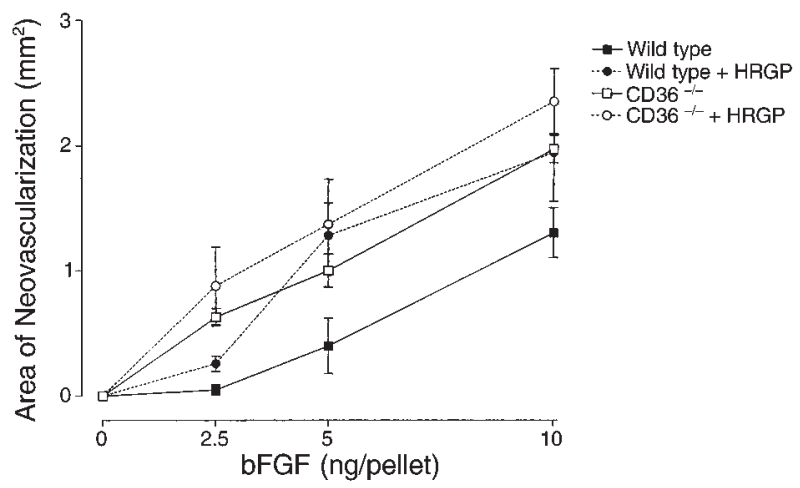

Figure 7

HRGP abrogates the effect of endogenous TSP in the corneal angiogenesis assay. Pellets containing bFGF at various doses with and without $50 \mathrm{ng}$ HRGP were implanted into the corneas of wild-type C57BL/ 6 (closed symbols) or CD36-null mice (open symbols), and the area of neovascularization was measured after 5 days. HRGP (filled circles) augmented the dose-dependent effect of bFGF (filled squares) in the wild-type mice $(P=0.0004$ by ANOVA). In CD36-null mice, there was an enhanced dose response to bFGF (open squares) $(P=0.01)$, but no effect was seen with the addition of HRGP (open circles). Results represent mean \pm SE of six or more replicates.

nous antiangiogenic activity mediated by TSP-1 or TSP-1-like ligands for the antiangiogenic receptor CD36. As expected, TSP-1 did not inhibit bFGFinduced angiogenesis in the CD36-null mice, confirming our previous findings (12) and providing further evidence that CD36 is necessary for the antiangiogenic activity of TSP-1.

These results were confirmed in an in vivo angiogenesis assay in which Matrigel impregnated with angiogenic growth factors was injected subcutaneously near the abdominal midline of mice. The resulting pellet was removed after 10 days, processed, and stained for histologic examination and blood vessel counts. As shown in Figure 8, we found that TSP-1 inhibited bFGF-induced blood vessel formation ( $14 \pm 8 \%$ of blood vessels $/ \mathrm{mm}^{2}$ seen with $\mathrm{bFGF}$ ), and that HRGP inhibited the antiangiogenic effect of TSP-1 ( $80 \pm 13 \%)$. These data demonstrate a physiologic role for HRGP in the modulation of the antiangiogenic activity of TSP-1 in two distinct in vivo angiogenesis models.

\section{Discussion}

The process of angiogenesis requires intricate control to allow for normal development and wound healing, and may lead to the progression and metastasis of tumors. Tumor masses that remain dormant and undetectable for years may suddenly acquire the ability to promote neovascularization, a process that has been termed "angiogenic switch" (34). Studies in murine models show that inhibition of angiogenesis may lead to regression of large tumor masses, suggesting that tumor angiogenesis is a dynamic process (35).
Recent attention has therefore focused on the identification and characterization of natural inhibitors of angiogenesis and their receptors, which provide attractive targets for therapeutic intervention.

Although several specific inhibitors of angiogenesis have been described, many of which have been found to be fragments of larger extracellular matrix-associated proteins $(7,36)$, TSP- 1 is the only one for which a receptor-ligand interaction has been well characterized. The chymotrypsin-resistant core of TSP-1, consisting of several properdin-like type I repeats, retains the antiangiogenic capacity of the intact molecule. Synthetic peptides derived from the type I domains have also been found to have potent antiangiogenic activity in vivo and in assays of EC function. Although TSP-1 interacts with a number of proteins, its antiangiogenic activity in vitro and in vivo is mediated by the glycoprotein receptor $\operatorname{CD} 36(12,13)$. Inhibition of the TSP-1-CD36 interaction with a blocking Ab to TSP1 has been shown to lead to more rapid re-endothelialization in a post-angioplasty setting, a process similar mechanistically to angiogenesis (37).

Although a number of interactions have been reported for HRGP, no in vivo physiologic function has been described previously for this abundant plasma protein. The structure of HRGP is unique, consisting of a number of discrete domains, including two cystatin-like domains at the $\mathrm{NH}_{2}$-terminus, a histidine-rich region, and two proline-rich domains (38). We have identified two regions in HRGP with significant homology to the TSP-1 binding site of CD36. These regions, known as CLESH-1 motifs, are conserved among members of the CD36 gene family and other TSP-1 binding proteins. Our studies show that binding of HRGP to TSP- 1 was mediated by the TSP type I repeats, the same sequence motifs responsible for antiangiogenic activity and CD36 binding. We demonstrated that HRGP inhibited the antiangiogenic activity of TSP-1 in vivo, and that HRGP augmented response to low doses of bFGF in a CD36-dependent manner, suggesting that HRGP antagonizes the effect of endogenous TSP-1.

\section{Table 1}

HRGP reverses the CD36-dependent antiangiogenic activity of TSP-1 in a corneal angiogenesis assay

\begin{tabular}{llcc}
\cline { 2 - 3 } Mouse & Pellet & $\begin{array}{c}\text { Area of neovascularization } \\
\pm \mathrm{SE}\left(\mathrm{mm}^{2}\right)\end{array}$ & $n$ \\
Wild type & bFGF & $2.35 \pm 0.10$ & 13 \\
& bFGF + TSP-1 & $0.51 \pm 0.21$ & 11 \\
& bFGF + TSP-1 + HRGP & $1.94 \pm 0.15^{\mathrm{A}}$ & 10 \\
& HRGP & $0 \pm 0$ & 4 \\
& HRGP \pm TSP-1 & $0.010 \pm 0.009$ & 4 \\
& bFGF \pm HRGP & $2.02 \pm 0.33$ & 4 \\
CD36-null & bFGF & $2.10 \pm 0.14$ & 6 \\
& bFGF + TSP-1 & $1.92 \pm 0.32$ & 8 \\
& bFGF + TSP-1 + HRGP & $2.30 \pm 0.19$ & 6 \\
& HRGP & $0.02 \pm 0.004$ & 4 \\
& HRGP \pm TSP-1 & $0.01 \pm 0.006$ & 4 \\
AP $=0.008$. & & &
\end{tabular}




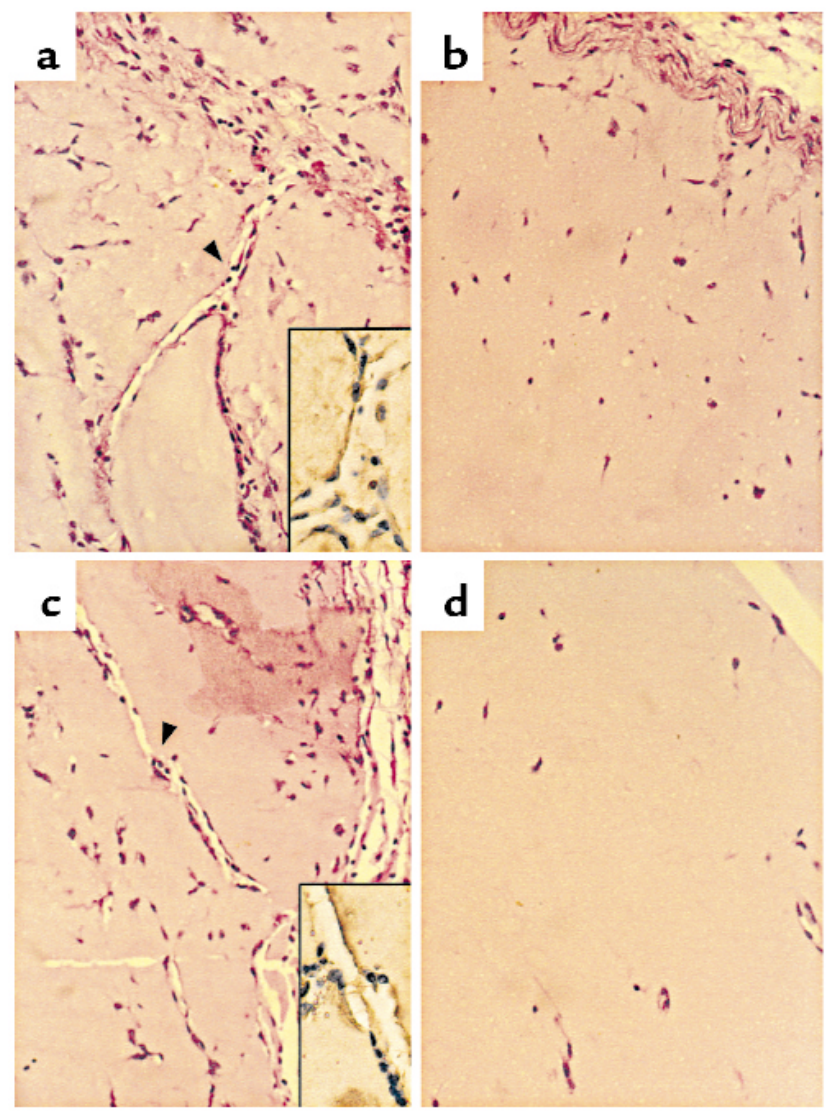

Figure 8

HRGP inhibits the antiangiogenic effect of TSP-1 in vivo. Matrigel injected subcutaneously into the midline of mice, and resulting plugs were harvested after 10 days. Plugs were fixed and embedded in paraffin. Blocks were then sectioned, stained with hematoxylin and eosin, and photographed $(\times 200)$. Representative images from four separate experiments are shown. (a) bFGF; (b) bFGF + TSP-1; (c) bFGF + TSP-1 + HRGP; (d) HRGP. Insets in a and $\mathbf{c}$ show immunohistochemical staining with anti-vWF Ab $(\times 400)$.

We therefore propose a model of the inhibition of the antiangiogenic effect of TSP-1 by HRGP (Figure 9). Interaction of the type I repeats of TSP-1 with the CLESH-1 domain of CD36 leads to a cascade of signaling events mediated by CD36. HRGP can interfere with the interaction of TSP-1 with CD36, and may therefore be a natural modulator of angiogenesis. The activity of HRGP may provide a mechanism by which tumors escape or become resistant to the antiangiogenic effects of TSP-1.

Previously described interactions of HRGP support the hypothesis that HRGP is an important modulator of angiogenesis. Because the binding of HRGP to heparin is $\mathrm{pH}$ dependent and increases at lower $\mathrm{pH}$, circulating HRGP may bind to glycosaminoglycans in matrix or on the surface of ECs, especially in areas of relative hypoxia and low $\mathrm{pH}$. HRGP in the matrix may also enhance angiogenesis by increasing fibrinolytic activity. Immobilized HRGP on a metal substrate (4) or in a trimolecular complex with TSP-1 and plasminogen accelerates plasminogen activation by tissue plasminogen activator. The rate of plasmin generation from the trimolecular complex was greater than from a bimolecular complex of TSPplasminogen, suggesting an important interaction of HRGP with plasminogen when both are complexed to TSP (39). Therefore, the organization of a protein complex in the matrix may play an important role in the regulation of proteolytic activity, and HRGP may serve as a natural, circulating mediator of the angiogenic switch.

Angiogenesis is particularly relevant to the pathogenesis of breast cancer. The degree of angiogenesis in breast cancer specimens has been shown to correlate with the rate of metastasis, and the level of angiogenesis in breast cancers has been shown to be an independent prognostic factor $(40,41)$. Primary human breast cancer cells express a number of proangiogenic factors such as bFGF and vascular endothelial growth factor, and TSP has long been known to be localized in the basement membrane and desmoplastic stroma of malignant breast epithelium (31). Using immunohistochemical studies of human breast cancer specimens, we found that HRGP colocalized with TSP-1 in the tumor matrix, and that this interaction masked the antiangiogenic epitope of TSP-1. Therefore, in areas where TSP-1 is an important inhibitor of angiogenesis, HRGP could serve as a modulator of TSP-1 activity, promoting angiogenesis. The biologic impact of these interactions may vary in different tumors as a consequence of the matrix components. Elucidation of these relationships may lead to new modalities of anticancer therapy.

\section{Figure 9}

Model: HRGP inhibits the antiangiogenic effect of TSP-1. Angiogenesis induced by bFGF (left) is inhibited by TSP-1 through the interaction of the TSP type I repeat with the $\mathrm{CLESH}-1$ domain of the signaling receptor CD36 (center). HRGP, which also contains the CLESH-1 motif, binds TSP-1, inhibiting the interaction of TSP- 1 with CD36, thereby inhibiting the antiangiogenic effect of TSP-1 (right).

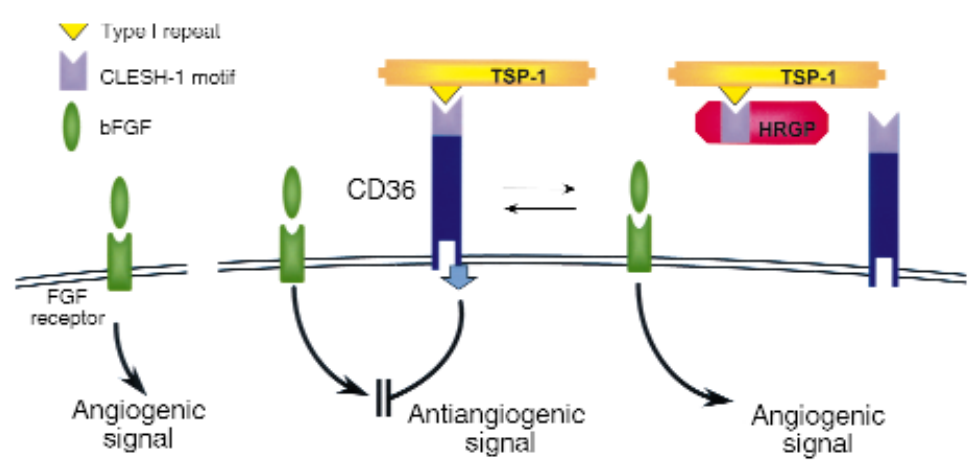




\section{Acknowledgments}

We thank Chun Jie Wang for technical assistance. Funding for this research was provided in part by the AMDeC Foundation of New York City through its Tartikoff/Perelman/EIF Fund for Young Investigators in Women's Cancers. R. Simantov is supported by Clinical Associate Physician Award M01 RR-00047 from the General Clinical Research Centers of the $\mathrm{NIH}$, and a Junior Faculty Scholars Award from the American Society of Hematology. This work was also supported by grants R01 HL-42540 (R.L. Silverstein) and R29 HL-58559 (M. Febbraio) from the NIH.

1. Leung, L. 1993. Histidine-rich glycoprotein: an abundant plasma protein in search of a function. J. Lab. Clin. Med. 121:630-631.

2. Leung, L.L. 1986. Interaction of histidine-rich glycoprotein with fibrinogen and fibrin. J. Clin. Invest. 77:1305-1311.

3. Silverstein, R.L., Nachman, R.L., Leung, L.L., and Harpel, P.C. 1985. Activation of immobilized plasminogen by tissue activator. Multimolecular complex formation. J. Biol. Chem. 260:10346-10352.

4. Borza, D.B., and Morgan, W.T. 1997. Acceleration of plasminogen activation by tissue plasminogen activator on surface-bound histidine-proline-rich glycoprotein. J. Biol. Chem. 272:5718-5726.

5. Angles-Cano, E., Gris, J.C., Loyau, S., and Schved, J.F. 1993. Familial association of high levels of histidine-rich glycoprotein and plasminogen activator inhibitor-1 with venous thromboembolism. J. Lab. Clin. Med. 121:646-653.

6. Leung, L.L., Nachman, R.L., and Harpel, P.C. 1984. Complex formation of platelet thrombospondin with histidine-rich glycoprotein. J. Clin. Invest. 73:5-12.

7. Good, D.J., et al. 1990. A tumor suppressor-dependent inhibitor of angiogenesis is immunologically and functionally indistinguishable from a fragment of thrombospondin. Proc. Natl. Acad. Sci. USA. 87:6624-6628.

8. Bagavandoss, P., and Wilks, J.W. 1990. Specific inhibition of endothelial cell proliferation by thrombospondin. Biochem. Biophys. Res. Commun. 170:867-872.

9. Tolsma, S.S., et al. 1993. Peptides derived from two separate domains of the matrix protein thrombospondin-1 have anti-angiogenic activity. $J$. Cell. Biol. 122:497-511.

10. Frazier, W.A., Dixit, V.M., Galvin, N.J., and Rotwein, P.R. 1987. Structure of human thrombospondin: complete amino acid sequence derived from cDNA. Semin. Thromb. Hemost. 13:255-260.

11. Iruela-Arispe, M.L., Bornstein, P., and Sage, H. 1991. Thrombospondin exerts an antiangiogenic effect on cord formation by endothelial cells in vitro. Proc. Natl. Acad. Sci. USA. 88:5026-5030.

12. Jimenez, B., et al. 2000. Signals leading to apoptosis-dependent inhibition of neovascularization by thrombospondin-1. Nat. Med. 6:41-48.

13. Dawson, D.W., et al. 1997. CD36 mediates the in vitro inhibitory effects of thrombospondin-1 on endothelial cells. J. Cell. Biol. 138:707-717.

14. Asch, A.S., et al. 1993. Analysis of CD36 binding domains: ligand specificity controlled by dephosphorylation of an ectodomain. Science. 162:1436-1440

15. Silverstein, R.L., and Nachman, R.L. 1987. Thrombospondin binds to monocytes-macrophages and mediates platelet-monocyte adhesion. $J$. Clin. Invest. 79:867-874.

16. Crombie, R., et al. 1998. Identification of a CD36-related thrombospondin 1-binding domain in HIV-1 envelope glycoprotein gp120: relationship to HIV-1-specific inhibitory factors in human saliva. J. Exp. Med. 187:25-35.

17. Pearce, S.F.A., Wu, J., and Silverstein, R.L. 1995. Recombinant GST/CD36 fusion proteins define a thrombospondin binding domain. Evidence for a single calcium-dependent binding site on CD36. J. Biol. Chem. 270:2981-2986.

18. Worley, K.C., Wiese, B.A., and Smith, R.F. 1995. BEAUTY: an enhanced BLAST-based search tool that integrates multiple biological information resources into sequence similarity search results. Genome Res. 5:173-184.

19. Huang, X.Q., Hardison, R.C., and Miller, W. 1990. A space-efficient algorithm for local similarities. Comput. Appl. Biosci. 6:373-381.

20. Polverini, P.J., Bouck, N.P., and Rastinejad, F. 1991. Assay and purification of naturally occurring inhibitor of angiogenesis. Methods Enzymol. 198:440-450.

21. Passaniti, A., et al. 1992. A simple, quantitative method for assessing angiogenesis and antiangiogenic agents using reconstituted basement membrane, heparin, and fibroblast growth factor. Lab. Invest. 67:519-528

22. Yamamoto, K., de Waard, V., Fearns, C., and Loskutoff, D.J. 1998. Tissue distribution and regulation of murine von Willebrand factor gene expression in vivo. Blood. 92:2791-2801.

23. Volpert, O.V., Lawler, J., and Bouck, N.P. 1998. A human fibrosarcoma inhibits systemic angiogenesis and the growth of experimental metastases via thrombospondin-1. Proc. Natl. Acad. Sci. USA. 95:6343-6348.

24. Kenyon, B.M., Browne, F., and D'Amato, R.J. 1997. Effects of thalidomide and related metabolites in a mouse corneal model of neovascularization. Exp. Eye Res. 64:971-978.

25. Rohan, R.M., Fernandez, A., Udagawa, T., Yuan, J., and D’Amato, R.J. 2000. Genetic heterogeneity of angiogenesis in mice. FASEB J. 14:871-876.

26. Iruela-Arispe, M.L., Lombardo, M., Krutzsch, H.C., Lawler, J., and Roberts, D.D. 1999. Inhibition of angiogenesis by thrombospondin-1 is mediated by 2 independent regions within the type 1 repeats. Circulation. 100:1423-1431.

27. Dawson, D.W., et al. 1999. Three distinct D-amino acid substitutions confer potent antiangiogenic activity on an inactive peptide derived from thrombospondin-1 type 1 repeat. Mol. Pharmacol. 55:332-338.

28. Vanguri, V.K., Wang, S., Godyna, S., Ranganathan, S., and Liau, G. 2000. Thrombospondin- 1 binds to polyhistidine with high affinity and specificity. Biochem. J. 347:469-473.

29. Clezardin, P., Frappart, L., Clerget, M., Pechoux, C., and Delmas, P.D. 1993. Expression of thrombospondin (TSP1) and its receptors (CD36 and CD51) in normal, hyperplastic, and neoplastic human breast. Cancer Res. 53:1421-1430.

30. Tuszynski, G.P., and Nicosia, R.F. 1994. Localization of thrombospondin and its cysteine-serine-valine-threonine-cysteine-glycine-specific receptor in human breast carcinoma. Lab. Invest. 70:228-233.

31. Wong, S.Y., Purdie, A.T., and Han, P. 1992. Thrombospondin and other possible related matrix proteins in malignant and benign breast disease. An immunohistochemical study. Am. J. Pathol. 140:1473-1482.

32. Crombie, R., and Silverstein, R. 1998. Lysosomal integral membrane protein II binds thrombospondin-1. Structure-function homology with the cell adhesion molecule CD36 defines a conserved recognition motif. J. Biol. Chem. 273:4855-4863.

33. Pijuan-Thompson, V., et al. 1999. Retinoic acid alters the mechanism of attachment of malignant astrocytoma and neuroblastoma cells to thrombospondin-1. Exp. Cell Res. 249:86-101.

34. Folkman, J., and Klagsbrun, M. 1987. Angiogenic factors. Science. 235:442-447.

35. O'Reilly, M.S., et al. 1994. Angiostatin: a novel angiogenesis inhibitor that mediates the suppression of metastases by a Lewis lung carcinoma. Cell. 79:315-328.

36. O'Reilly, M.S., et al. 1997. Endostatin: an endogenous inhibitor of angiogenesis and tumor growth. Cell. 88:277-285.

37. Chen, D., et al. 1999. Antibody blockade of thrombospondin accelerates re-endothelialization and reduces neointima formation in ballooninjured rat carotid artery. Circulation. 100:849-854.

38. Koide, T., and Odani, S. 1987. Histidine-rich glycoprotein is evolutionarily related to the cystatin superfamily. Presence of two cystatin domains in the N-terminal region. FEBS Lett. 216:17-21.

39. Silverstein, R.L., Leung, L.L., Harpel, P.C., and Nachman, R.L. 1985. Platelet thrombospondin forms a trimolecular complex with plasminogen and histidine-rich glycoprotein. J. Clin. Invest. 75:2065-2073.

40. Weidner, N., et al. 1992. Tumor angiogenesis: a new significant and independent prognostic indicator in early-stage breast carcinoma. J. Natl. Cancer Inst. 84:1875-1887.

41. Weidner, N., Semple, J.P., Welch, W.R., and Folkman, J. 1991. Tumor angiogenesis and metastasis: correlation in invasive breast carcinoma. N. Engl.J. Med. 324:1-8. 